\title{
Automatic Segmentation of the Vertebrae and the Transverse Processes Regions on CT Images
}

\author{
Ran DONG ${ }^{\text {a,b*, }}$ Masashi KONDO ${ }^{\mathrm{a}}$, Joo Kooi TAN ${ }^{\mathrm{a}}$, \\ Yihong $\mathrm{ZHAO}^{\mathrm{b}}$, Youwei $\mathrm{ZHAO}^{\mathrm{b}}$, Hyoungseop $\mathrm{KIM}^{\mathrm{a}}$ \\ aKyushu Institute of Technology, 1-1, Sensui, Tobata, Kitakyushu 804-8550, Japan \\ bYangzhou University, 196, Huayangxilu, Hanjiang, Yangzhou 225127, China \\ *Corresponding Author: p344234@ mail.kyutech.jp
}

\begin{abstract}
CT (Computed Tomography) scanners, that are experiencing durative innovation in virtue of development of computer technology, are diffusely employed by radiologists in hospitals. Accompanied with these advanced medical equipment, CAD (Computer-aided Diagnosis) system has been proposed in the meantime aimed to assist radiologists to judge illnesses fleetly and precisely. Furthermore, segmentation of the region of interest, as one of the momentous steps in CAD system, can directly impact proceedings of image processes.

In this paper, automatic segmentation method to extract human vertebra and transverse process structures from CT images is put forward. To begin with, we actualize the initial extraction of spinal region by means of reduction of searching area. After that, labeling algorithm is applied to track vertebrae and transverse processes boundaries in spine with binary images which have underwent denoising with morphological processes. Ultimately, we segment vertebrae and transverse processes from original CT images with region grow algorithm. As experimental results, we acquire 98.74[\%] of average TP (True-Positive), 2.34[\%] of average FP (False-Positive) for vertebrae, 92.79[\%] of average TP,
\end{abstract}

$3.14[\%]$ of average FP for left transverse processes, and 90.69[\%] of average TP, 2.50[\%] of average FP for right transverse processes based on 10 slices with $1[\mathrm{~mm}]$ thickness.

Keywords: Binary, Morphological processes, Labeling algorithm, Region recovery algorithm, Segmentation.

\section{Introduction}

CAD (Computer-aided Diagnosis) has become one of main investigative subjects in medical imaging and diagnostic radiology. However, the performance level of output is still demanded to be heightened with automatic computer detection because of higher identification requirements than before ${ }^{(1)}$. Moreover, as one of the main components of image processes and computer vision, segmentation also shows its prosperous gesture in clinical diagnosis. For instance, Aslan et al. ${ }^{(2,3)}$ presented a 3D segmentation framework of VBs (Trabecular Bones) in CT images, and applied matched filter, graph cuts and volume growing approaches to separate VBs, respectively. Preliminary result with approximate 2[\%] segmentation error was achieved for 16 data sets. Song et al. ${ }^{(4)}$ developed a computer-aided method to segment liver from CT images 
employing a novel pre-processor and adaptive FMM (Fast Marching Method). Tan et al. ${ }^{(5,6)}$ presented a segmentation method to the whole vertebrae with syndesmophytes utilizing a 3D multi-scale cascade of successive level sets.

They sought out seed placement manually, and validated results based on synthetic and real data. Ibanez et al. (7) implemented this technique with the open library ITK functions. In addition, updated methods have been proposed to extract ventricle, heart, spine and other organs of human bodies ${ }^{(8-10)}$, and the references.

But many segmentation methods, which have been proposed until now, are faced with limitation to realize intelligence of image processes as results of relative lower universal property of them, changeable tissue features without unified discipline and the complexity of the fine structures ${ }^{(11)}$.

In this paper, an automatic method to segment vertebrae and transverse processes from CT images is developed. What is different from other methods already proposed is that we not only targets, but also extract them from original images and display them in different background with black color. Concretely, Section 2 describes main procedures including preprocesses of original images, detection of target boundaries with label algorithm, and final segmentation of vertebrae and transverse processes on account of region recovery algorithm. Section 3 shows the experimental results based on 10 slices with $1[\mathrm{~mm}]$ thickness. Section 4 states our conclusions.

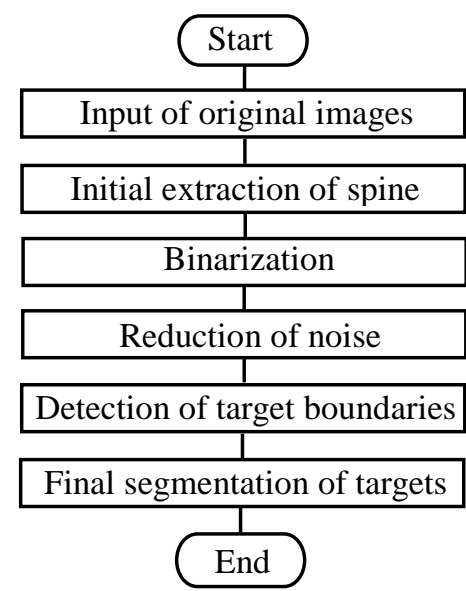

Fig.1 Segmentation of vertebrae and transverse processes

\section{Methods}

Overall flow chart about main steps of segmentation is constructed (see Fig.1). Every vital part, enriched by corresponding knowledge, will be illustrated with intermediate results.

\subsection{Initial extraction of spine}

Spinal region, which is surrounded by other organs of patient's anocoelia, should be switched from global scale image (Fig.2 (a)) into smaller square with $147 * 150 \mathrm{~mm}^{2}$ (Fig.2 (b)) after input of original images. Because it can not only cut down time consumption of following steps, but also abate effects of some other organs that have the same gray with spine and could interfere further segmentation.

\subsection{Binarization}

We prefer to translate colorful and gray level images into binary images before conducting analyses to objects. However, the certain reality is that omnipotent method to binary has not been doped out as the result of huge difference between changeable distribution rules of destiny. Chen (12) applied Otsu method to do binaziration for the global of plate numbers, as well as Liu. ${ }^{(13)}$ Who attempted to adopt Otsu method in local areas and harvested better results with distinct boundaries.

In this part, we plot the histogram of gray distribution in the smaller square aimed to grasp destiny features of spine and select suitable threshold for binarization. Also, we experiment sample images of patients with Otsu method to find out the threshold (See Fig.3 (a)), but there are much noise appearing after binarization. So we present an adaptive method and get better binary results. As shown in Fig.3 (b), we obtain clearer outline to perform further segmentation despite relative lesser noise still remain and some spinal regions disappear.

\subsection{Detection of target boundaries}

There are three targets that are left transverse process, 
vertebra and right transverse process from the left side to the right side of Fig.2 (b) respectively. We perform morphological processes such as erosion and dilation to reduce noise in binary results before detection, and then image anamorphose caused by noise in previous images can be obviously ameliorated with a threshold. What need to pay attention to are that the threshold is different from the one that we set in binarization and we choose it by destiny features. After that, these objects will be labeled by different numbers with steps as follows:

Step1: Input binary results and check pixels in images with TV raster scan to mark them with identifier, and then distribute them into the same group.

Step2: If the gray of $x_{0}$ (Fig.4) equals 0,return to Step1.

Step3: If $x_{3}$ and $x_{5}$ (Fig.4) are all marked with apoise numbers, label $x_{0}$ with the smaller number in $x_{3}$ and $x_{5}$.

Step4: If scanning is not terminate, return to Step1.

Step5: Mark all pixels with the smallest number in the same region.

Finally, we receive all boundaries of left transverse processes, vertebrae and right transverse processes with steps above, and one of the detected results with other organs in sample image as background is shown in Fig.5. The boundaries in figure are depicted with red line.

\subsection{Final segmentation of targets}

No sooner is the application of object region recovery algorithm on the basis of boundaries that we acquire from Fig.5, than the ultimate segmentation outcomes of vertebrae and transverse processes in 10 slices can be acquired.

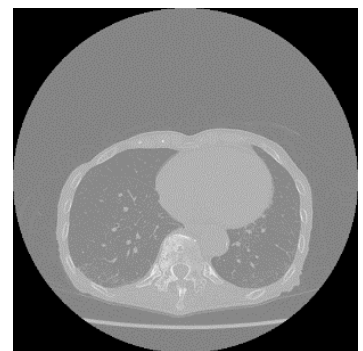

(a)

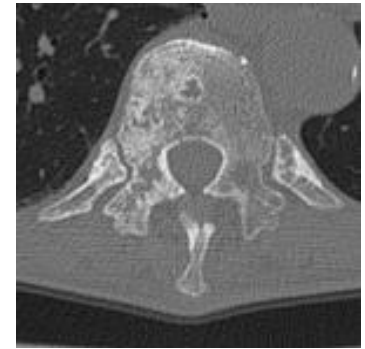

(b)
Fig.2 (a) Global scale image. (b) Smaller square of spine.

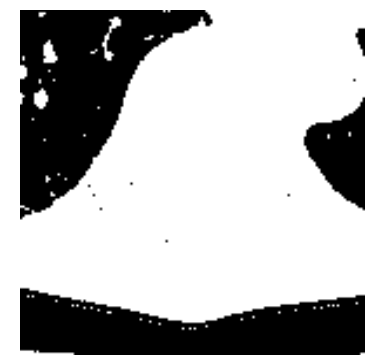

(a)

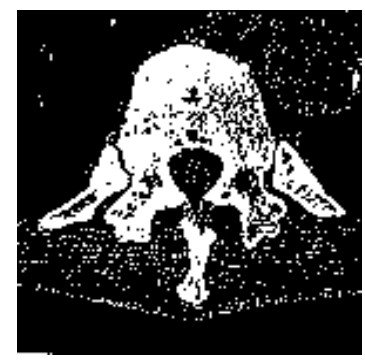

(b)
Fig.3 (a) Binarization with Otsu method. (b) Binarization with proposed method.

\begin{tabular}{|c|c|c|}
\hline$x_{4}$ & $x_{3}$ & $x_{2}$ \\
$(\mathrm{i}-1, \mathrm{j}-1)$ & $(\mathrm{i}-1, \mathrm{j})$ & $(\mathrm{i}-1, \mathrm{j}+1)$ \\
\hline$x_{5}$ & $x_{0}$ & $x_{1}$ \\
$(\mathrm{i}, \mathrm{j}-1)$ & $(\mathrm{i}, \mathrm{j})$ & $(\mathrm{i}, \mathrm{j}+1)$ \\
\hline$x_{6}$ & $x_{7}$ & $x_{8}$ \\
$(\mathrm{i}+1, \mathrm{j}-1)$ & $(\mathrm{i}+1, \mathrm{j})$ & $(\mathrm{i}+1, \mathrm{j}+1)$ \\
\hline
\end{tabular}

Fig.4 8-neighbor of the pixel $\mathrm{x}_{0}=(\mathrm{i}, \mathrm{j})$

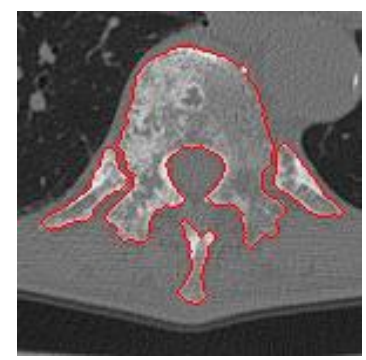

Fig.5 Vertebrae and transverse processes boundaries

\section{Experimental Results}

The cardinal goal of our experiment is segmentation of vertebrae and transverse processes. We segment objects with proposed method in 30 seconds successfully, and final results are shown in Fig.6. Results with black background retain almost all intensity features in spite of ambiguous boundary problems of original CT images.

We evaluate the performance with true-positive in Eq. (1) and false-positive in Eq. (2). The detailed descriptions of these two criteria are as follows. 


$$
\begin{aligned}
& T P=\frac{n(A \cap B)}{n(A)} \times 100[\%] \\
& F P=\frac{n(C)}{n(B)} \times 100[\%]
\end{aligned}
$$

where $A, B, C$ and $n$ are relative more precise segmented region, extracted region with our method, over-extracted region with our method, and pixel value respectively. TP represents the overlap degree of the extracted region against more accurate outcome while FP expresses the percentage of over-extracted region in our experiment.

As evaluation results (Table 1-2), we get every TP and FP of 10 slices CT image with 1 [mm] thickness. In addition, 98.74[\%] of average TP and 2.34[\%] of average FP are for vertebrae. 92.79[\%] of average TP and 3.14[\%] of average FP are for left transverse processes. 90.69[\%] of average TP and $2.50[\%]$ of average $\mathrm{FP}$ are for right transverse processes.

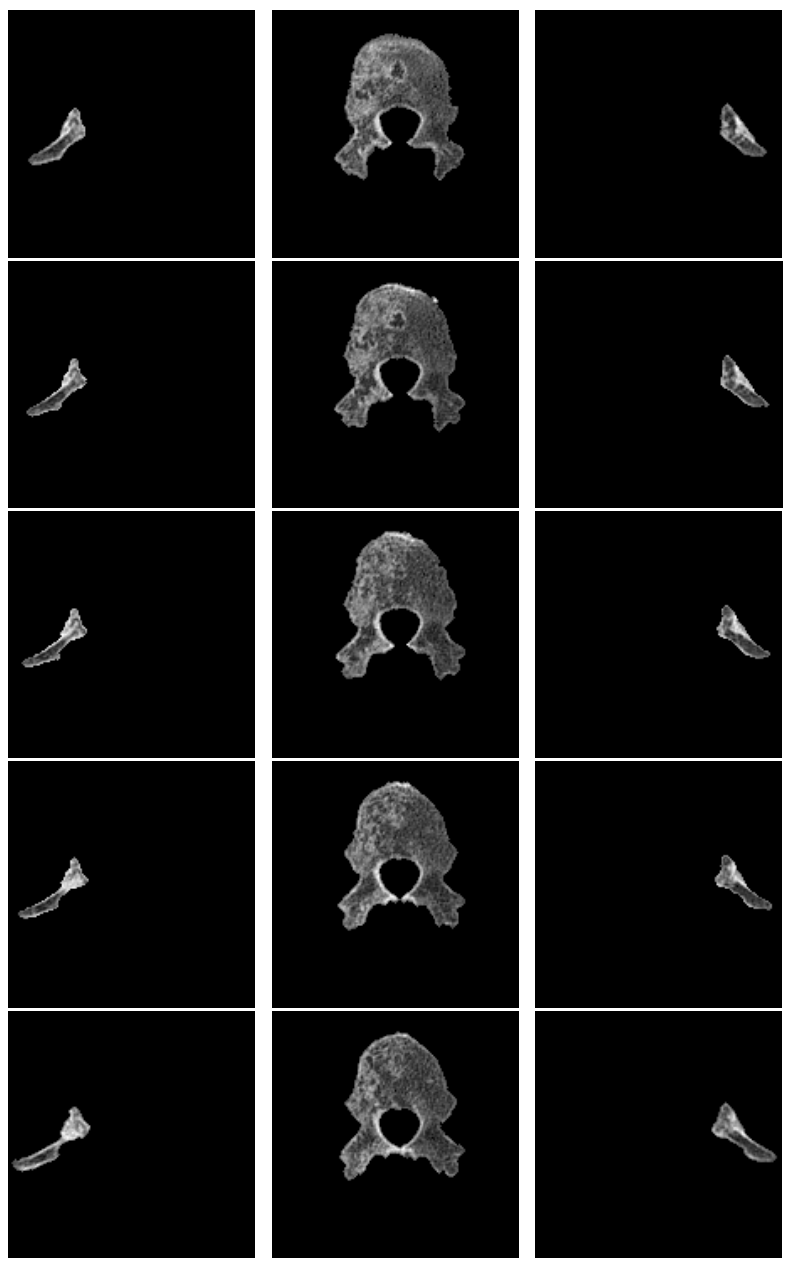

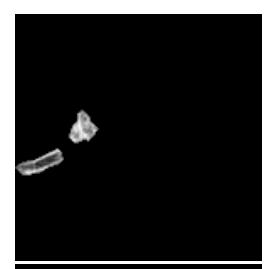
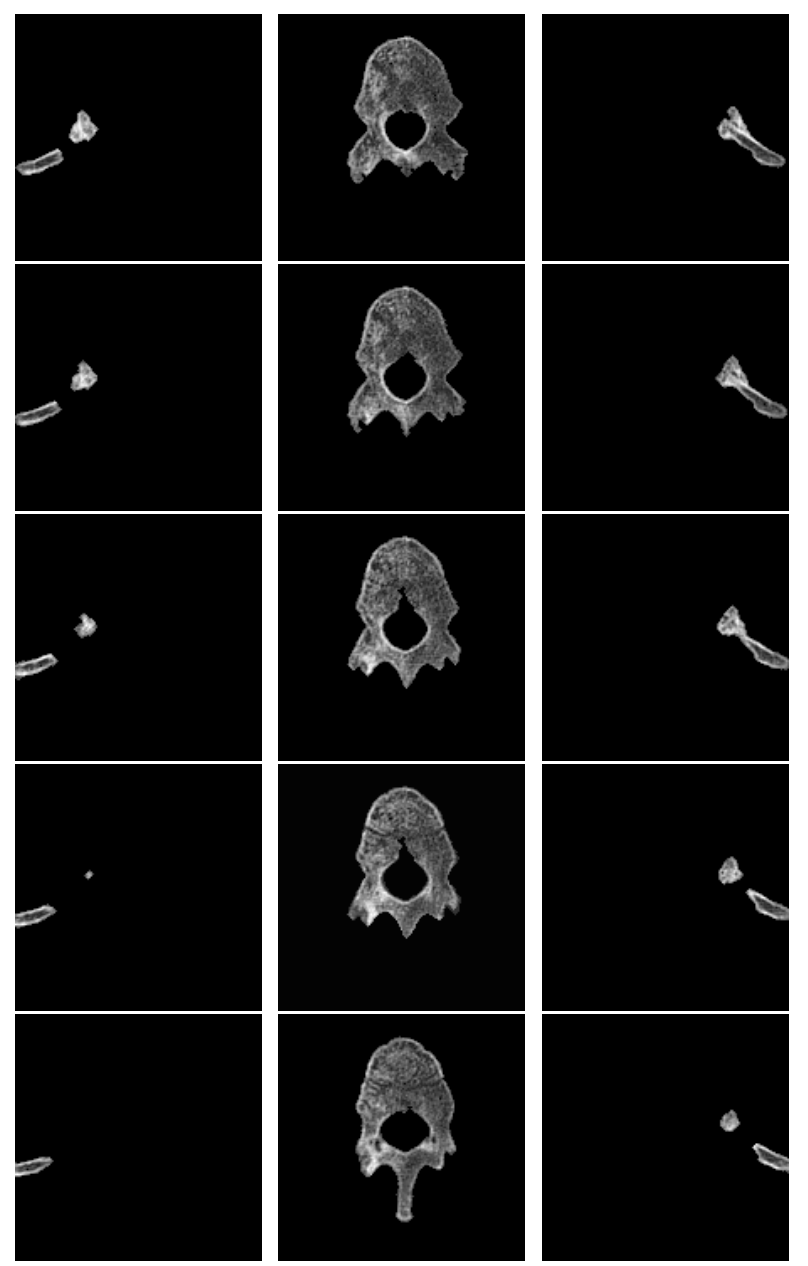

(a)

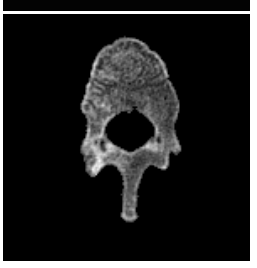

(b)

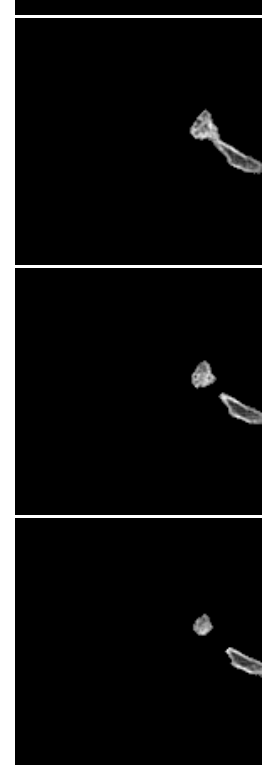

(c)
Fig.6 (a) Left transverse process. (b) Vertebra. (c) Right transverse process.

Table 1. True-Positive

\begin{tabular}{|c|c|c|c|}
\hline Case & Vertebrae & $\begin{array}{c}\text { Light } \\
\text { Transverse } \\
\text { Processes }\end{array}$ & $\begin{array}{c}\text { Right } \\
\text { Transverse } \\
\text { Processes }\end{array}$ \\
\hline 1 & $98.06 \%$ & $88.68 \%$ & $88.64 \%$ \\
\hline 2 & $99.27 \%$ & $86.61 \%$ & $81.62 \%$ \\
\hline 3 & $98.87 \%$ & $81.59 \%$ & $79.87 \%$ \\
\hline 4 & $98.91 \%$ & $81.79 \%$ & $78.54 \%$ \\
\hline 5 & $99.35 \%$ & $100.00 \%$ & $98.95 \%$ \\
\hline 6 & $99.09 \%$ & $98.34 \%$ & $96.39 \%$ \\
\hline 7 & $98.41 \%$ & $98.96 \%$ & $96.79 \%$ \\
\hline 8 & $97.53 \%$ & $99.78 \%$ & $92.90 \%$ \\
\hline 9 & $98.52 \%$ & $98.66 \%$ & $94.93 \%$ \\
\hline 10 & $99.36 \%$ & $93.33 \%$ & $98.28 \%$ \\
\hline Average & $98.74 \%$ & $92.79 \%$ & $90.69 \%$ \\
\hline
\end{tabular}


Table 2. False-Positive

\begin{tabular}{|c|c|c|c|}
\hline Case & Vertebrae & $\begin{array}{c}\text { Light } \\
\text { Transverse } \\
\text { Processes }\end{array}$ & $\begin{array}{c}\text { Right } \\
\text { Transverse } \\
\text { Processes }\end{array}$ \\
\hline 1 & $3.56 \%$ & $0.00 \%$ & $2.99 \%$ \\
\hline 2 & $3.18 \%$ & $1.06 \%$ & $1.06 \%$ \\
\hline 3 & $1.83 \%$ & $0.00 \%$ & $0.00 \%$ \\
\hline 4 & $1.09 \%$ & $0.00 \%$ & $0.00 \%$ \\
\hline 5 & $1.99 \%$ & $3.39 \%$ & $3.29 \%$ \\
\hline 6 & $1.88 \%$ & $4.44 \%$ & $2.51 \%$ \\
\hline 7 & $1.94 \%$ & $5.17 \%$ & $3.69 \%$ \\
\hline 8 & $1.34 \%$ & $5.99 \%$ & $7.10 \%$ \\
\hline 9 & $5.89 \%$ & $7.77 \%$ & $3.74 \%$ \\
\hline 10 & $0.72 \%$ & $3.61 \%$ & $0.58 \%$ \\
\hline Average & $2.34 \%$ & $3.14 \%$ & $2.50 \%$ \\
\hline
\end{tabular}

\section{Conclusions}

In order to segment vertebrae and transverse processes from CT images, we conduct binirization with adaptive threshold at first. Then, labeling algorithm and region recovery algorithm based on object boundaries are wielded in this paper. As experimental results within 30 seconds, we obtain encouraging segmentation of each target which has vivid contour and conserves almost all features in original images. It also provides ponderable and reliable foundation for following processes in CAD system. Meanwhile, the evaluations reveal that 98.74[\%] of average TP and 2.34[\%] of average FP for vertebrae are also relative progressive compared with experimental results of Aslan ${ }^{(2,3)}$.

However, TP of transverse processes share relative lower percentage in case 1-5 of Fig.7, as well as the contrary situation that case 7-10 own relative higher FP in Fig.8. We attribute these defects to lack of partial information of boundaries due to erosion and dilation operations in part 2.3.

\section{Acknowledgment}

The authors would like to thank MD.Takatoshi Aoki for providing the data.

\section{References}

(1) K. Doi: "Computer-aided Diagnosis in Medical Imaging: Historical review, current status and future potential", Computerized Medical Imaging and Graphics, vol.31, no 4-5, pp.198-211, 2007

(2) Melih S.Aslan, Asem Ali, and Ben Arnold: "Segmentation of Trabecular Bones from Vertebral Bodies in Volumetric CT Spine Images", The International Conference on Image Processing, pp.3385-3388, 2009

(3) Melih S.Aslan, Asem Ali, Aly A: "3D Vertevral Body Segmentation Using Shape Based Graph Cuts", International Conference on Pattern Recognition, pp.3951-3954, 2010

(4) Xiao Song: "Automatic liver Segmentation from CT Images Using Adaptive Fast Marching Method", International Conference on Image and Graphics, pp.897-900, 2015

(5) S.Tan: "Computer aided evaluation of Ankylosing Spondylitis", IEEE International Symposium on Biomedical Imaging, pp.339-342, 2006

(6) S.Tan: "Computer aided Evaluation of Ankylosing Spondylitis Using High-Resolution CT", IEEE Transaction on Medical Imaging, vol.27, no.9, pp.1252-1267, 2008

(7) L. Ibanez, W. Schroeder, and J. Cates: "The ITK software guide Kitware Inc", 2003

(8) Steven C: "Multistage Hybrid Active Appearance Model Matching: Segmentation of Left and Right Ventricles in Cardiac MR Images", IEEE Transactions on Medical Imaging, vol.20, no.5, pp.415-423, 2001

(9) Olivier Ecabert: "Automatic Model-Based Segmentation of the Heart in CT images", IEEE Transactions on Medical Imaging, vol.27, no.9, pp.1189-1201, 2008 
(10) Samuel Kadoury: "Spine Segmentation in Medical Images Using Manifold Embedding and Higher-Order MRFs", IEEE Transactions on Medical Imaging, vol.32, no.7, pp.1227-1238, 2013

(11) Ming. Qiu and Erhu. Zhang: "Methods of medicalimage segmentation", Computer Engineering and Design, vol.26, no.6, pp.1557-1559, 2005

(12) Si Chen: "The binarization of Plate Number with Otsu Method based on Matlab", Journal of Changchun Normal University, vol.31, no.3, pp.33-35, 2012

(13) Jihong Liu, and Chengyuan Wang: "An Algorithm for Image Binary with Adaptive Threshold", Chinese Control and Decision Conference, 2009 